\title{
A multi-scale point of view on the structure-property relationships of A15 superconductors
}

\author{
Yanlong Ding • Min Pan • Shuiquan Deng • \\ Yong Zhao
}

Received: 3 December 2013/Revised: 9 February 2014 / Accepted: 16 March 2014/Published online: 2 April 2014

(C) The Author(s) 2014. This article is published with open access at Springerlink.com

\begin{abstract}
Nb}_{3} \mathrm{Sn}$ and other $\mathrm{A} 15$ members have been widely applied in nuclear power, nuclear magnetic resonance, and high-energy particle accelerators for their high critical current density $\left(J_{\mathrm{c}}\right)$ and upper critical field $\left(B_{\mathrm{c} 2}\right)$. There have been comprehensive and intensive studies on the applications, the fundamental lattice dynamic and electronic properties, etc., of A15 superconductors. Various reviews on the preparations, structures, and properties have already been written in the last few years. Nevertheless, on account of the large amount of existing facts and views, a coherent view on the relations between the structures and properties has not appeared to unify the facts. This article sketches a multi-scale point of view on the relations between the multiscale structures and the corresponding properties.
\end{abstract}

Keywords Multi-scale $\cdot$ A15 compounds - Structure · Properties

\section{Introduction}

The first A15 compound, $\mathrm{V}_{3} \mathrm{Si}$ [1], was discovered by Hardy and Hulm in 1953. Since then a large number of compounds in this family have been prepared and characterized [2-5]. Among the important discoveries, the superconducting properties of some members such as $\mathrm{Nb}_{3} \mathrm{Sn}, \mathrm{Nb}_{3} \mathrm{Ge}$, etc., have received increasing attention. Although many other high $T_{\mathrm{c}}$ superconductors have also been discovered in the last few

Y. Ding $\cdot$ M. Pan $\cdot$ S. Deng $(\bowtie) \cdot$ Y. Zhao

Key Laboratory of Magnetic Levitation Technologies and Maglev Trains, Ministry of Education of China, and

Superconductivity and New Energy R\&D Center, Southwest

Jiaotong University, Chengdu 610031, China

e-mail: s.deng@swjtu.edu.cn years, they have never received large-scale applications due to their various technical problems. In fact, nowadays - in almost everywhere - a high magnetic field is necessary, so A15 superconductors are used. With the pressing energy problem, $\mathrm{Nb}_{3} \mathrm{Al}$ and $\mathrm{Nb}_{3} \mathrm{Sn}$ have been suggested as the superconductors for creating the high magnetic field to control the plasma in the International thermonuclear experimental reactor (ITER). In addition, the large amount of need for superconductors in the superconducting maglev (SCMaglev) [6] vehicle has also stimulated studies for developing new techniques for manufacturing stable and durable superconductors. In this regard, the conventional A15 superconductors have their own advantages. The main advantages of A15 superconductors are their overall best merit factors. For example, $\mathrm{Nb}_{3} \mathrm{Al}$ has a very high upper critical field $\left(B_{\mathrm{c} 2}\right)$, $\sim 30 \mathrm{~T}$ at $4.2 \mathrm{~K}$, and very good mechanical property which is important in actual applications. As the studies in this field are rather comprehensive and intensive, a large amount of facts and knowledge have been accumulated, ranging from microscopic electronic structures to macroscopic industry preparations. As diversified views exist for many experimental results at different length and energy scales, it is obvious that a unified and multi-scale point of view is necessary to summarize and analyze the existing results and conclusions. This work attempts to sketch such a frame to understand the relationship between the structures and properties of A15 compounds.

\section{Atomic scales}

\subsection{Crystal structure}

The A15 compounds with the chemical formula $\mathrm{A}_{3} \mathrm{~B}$ (the space group $P m \overline{3} n$ ) have the same crystal structure as 
shown in Fig. 1. The structure belongs to a simple cubic crystal system. Each unit cell contains six A-type atoms which lie on the surface of the unit cell and form chains along the axis directions, respectively. On each chain, the A-type atoms distribute equidistantly. The two B-type atoms occupy the corners and the center of a unit cell.

In principle, a crystallographic structure at the atomic scale is decided by the structure of subatomic scale, namely the electronic structure. In the case of A15 compounds, although the electronic structure and phonon spectrum have been intensively studied, a direct link between the electronic structure and crystallographic structure has not been established. The structures at this two length scales are usually studied separately.

\subsection{Phenomena related to the structure at atomic scale}

Some properties of a material are highly related to the structure at the atomic scale. The studies on the defects and their influences are crucial for practical applications of a material. Sweedler et al. [7] have studied the neutron irradiation effect on the superconducting properties of A15 compounds. As shown in Fig. 2, the superconducting transition temperature $\left(T_{\mathrm{c}}\right)$ is dramatically reduced with the irradiation of neutron. Although such kind of phenomenological relation as shown in Fig. 2 is established, the structural damages due to irradiation have remained unknown. Brown et al. [8] have also studied the influence of neutron irradiation on the critical current density of $\mathrm{Nb}_{3} \mathrm{Sn}$ (Fig. 3). As can be seen from Fig. 3, the critical currents are strongly affected by the field and irradiation dose. At high field, the change rate of critical current $\left(\Delta J_{\mathrm{c}}\right)$ $J_{\mathrm{c} 0} \equiv\left(J_{\mathrm{c}}-J_{\mathrm{c} 0}\right) / J_{\mathrm{c} 0}$, where $J_{\mathrm{c} 0}$ is the unirradiated $\left.J_{\mathrm{c}}\right)$ increases with the increase of the irradiation dose. At low field, the change rate of critical current decreases with the increase of the irradiation dose.

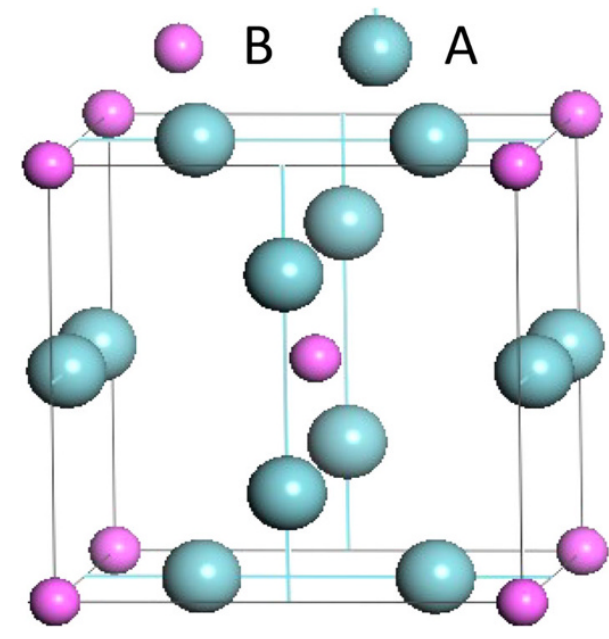

Fig. 1 The unit cell structure of A15 compound, $A_{3} B$
As the structural details after the irradiation have remained unknown, many experimentally established relations, as shown in Figs. 2 and 3, have not been well understood. In some studies, the reduction of the transition temperature $\left(T_{\mathrm{c}}\right)$ of A15 superconductors is attributed to the static disorder. It is thought that the high-energy irradiation induces "antisite defects" (A-type atom occupies the site of B-type atom and vice versa) [9]. These defects reduce the Bragg-Williams long-range-order parameter $\mathrm{S}$ [10] of the A15 superconductors. A possible consequence of such homogeneous defects is the reduction of the density of states at the Fermi level, DOS $\left(E_{\mathrm{F}}\right)$. Other possible effects of irradiation are the creation of disordered microregions, i.e., the so-called inhomogeneous defects, or the static displacement of atoms. Such defects may also cause the decrease of $T_{\mathrm{c}}$ by the proximity effect [11]. Although a wealth of experimental data has been obtained by various experimental means, the connection between the properties and the defected structures at the atomic scale has not been established due to lack of both the knowledge of the structures and the reliable microscopic models.

\section{Electronic structure}

Although in principle it is the structure at smaller scale which decides that at larger scale, the actual studies are performed in a reversed direction. This is because that humankind lives in a macroscopic world and thus our knowledge on the nature is established from macroscopic scale down to the microscopic scale in one direction and up to the cosmological scale in the other direction. The case of A15 compounds follows the above general principle. Because of the identical crystallographic structure, all A15 compounds have very similar electronic structures which differ only in details resulting from either different A- or B-type atoms. In Fig. 4, we have shown the electronic density of states (DOS) and the band structure of $\mathrm{Nb}_{3} \mathrm{Al}$ as

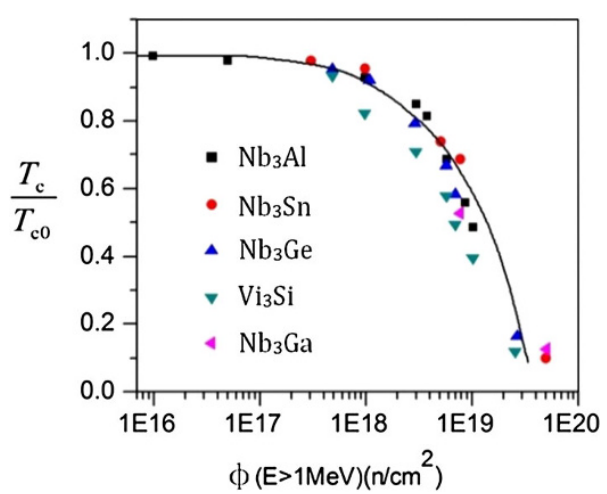

Fig. $2 T_{\mathrm{c}}$ as a function of neutron fluence $(E \leq 1 \mathrm{MeV}$, $T_{\text {irr }} \leq 150{ }^{\circ} \mathrm{C}$ ) 


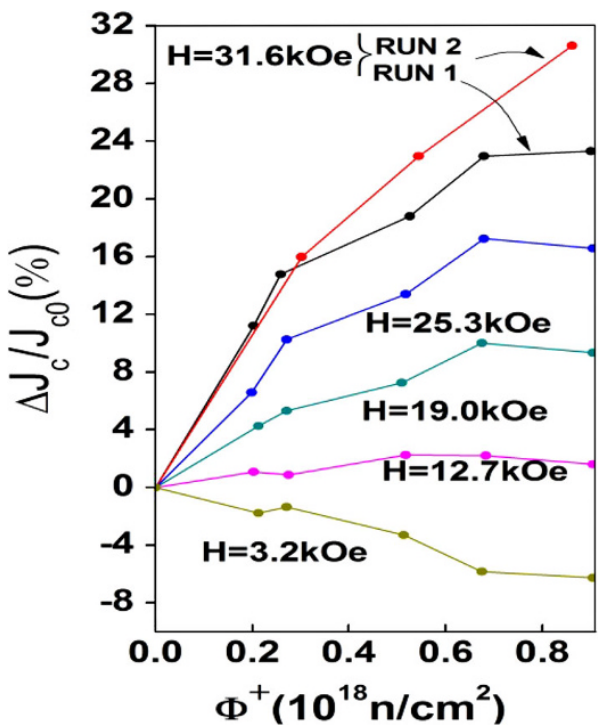

Fig. 3 The change rate of critical current as a function of field and irradiation dose

an example. The DOS has a sharp peak near the Fermi level. The states around the Fermi level are mainly contributed by the $\mathrm{Nb} d$ states. The lowest peak at about $-5.6 \mathrm{eV}$ is contributed mainly by the $s$ states of B-type atoms [12]. These states form the lowest dispersion-less non-bonding energy bands. The bands above the Fermi level are mainly contributed by the $p$ states of the B atoms. The coexistence of the very flat bands and steep bands around the Fermi level indicates that the A15 superconductors are essentially of "flat/steep" band superconductors [13]. The consequence of this latest observation [14] is worth of further studies.

\section{Properties related to mesoscopic structure}

For practical applications, the critical current density $\left(J_{\mathrm{c}}\right)$ and the upper critical field $\left(H_{\mathrm{c} 2}\right)$ are crucial parameters. There have been a number of established experimental facts that indicate the notable dependence of $J_{\mathrm{c}}$ on the grain size of the sample [15-17]. It has also been found that the pinning force shows also some dependence on the grain size and grain boundary. In addition, the critical superconducting properties have also been found to be affected by the stacking faults [18]. It should be noted that knowledge of the grain size, grain boundary, etc., provides structural information possibly ranging from nano to mesoscopic length scale. Because of the inadequacy of the structural information and the lack of reliable model at these length scales, the explanations on the phenomenological structure-properties relations as established by experiments are not conclusive [18-20].
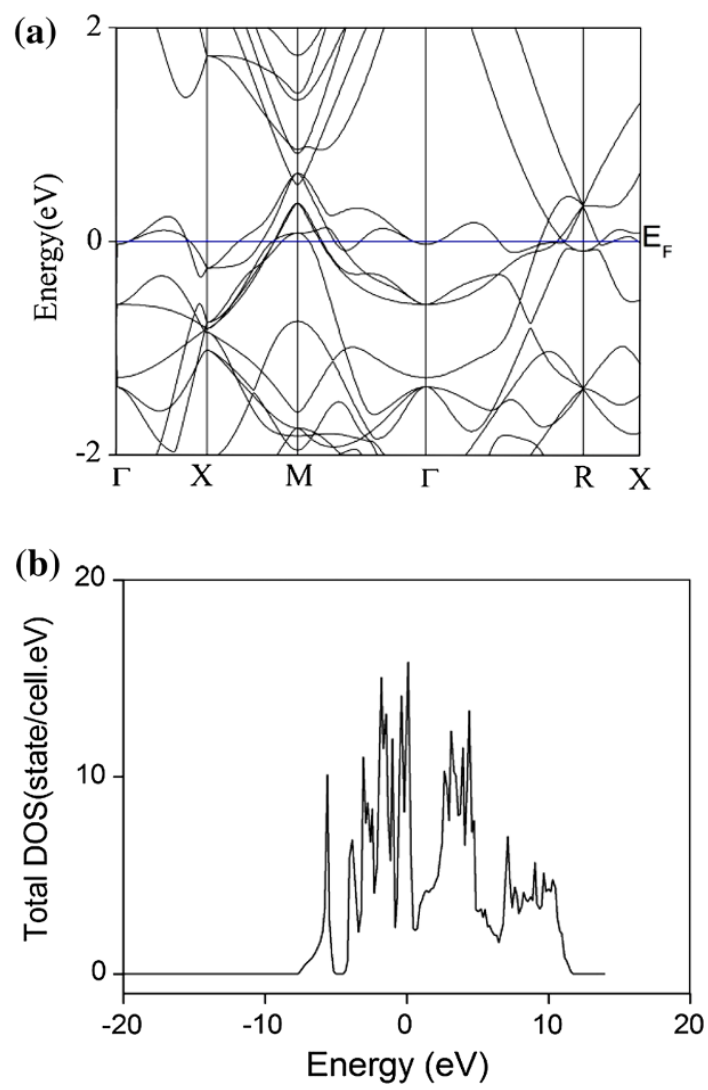

Fig. 4 a The band structure of $\mathrm{Nb}_{3} \mathrm{Al}$. b The total density of states of $\mathrm{Nb}_{3} \mathrm{Al}$

\section{Macroscopic structure and properties}

It is evident that to apply a stress on a material, its macrostructure will be changed in the form of strain, deformation, or cracks, etc. [21, 22]. For A15 superconductors, indirect indicators for such changes are superconducting critical parameters such as $J_{\mathrm{c}}, H_{\mathrm{c} 2}$, and $T_{\mathrm{c}}$. Figure 5 shows the effect of uniaxial strain on the upper critical field of A15 superconductors [23]. As shown in Fig. 5, although the crystal structures, electronic structures, etc., of A15 compounds are very similar, their critical field and critical current density have very different responses on the stress. Taylor and Hampshire [24] and Markiewicz [25] have attributed the effects of the uniaxial strain on the superconducting critical parameters to the changes of the phonon spectrum, rather than to the electronic density of states. In contrast, other researchers [26, 27] have attributed these effects mainly to the electronic properties. The problem of such studies is that the direct effect of the macroscopically applied stress has not been known with regard to the structures at macroscopic, mesoscopic, nanoscopic, and atomic length scales. Such information has essentially been taken as assumptions in the above-mentioned studies. A recent work [27] which has taken electronic and phonon 


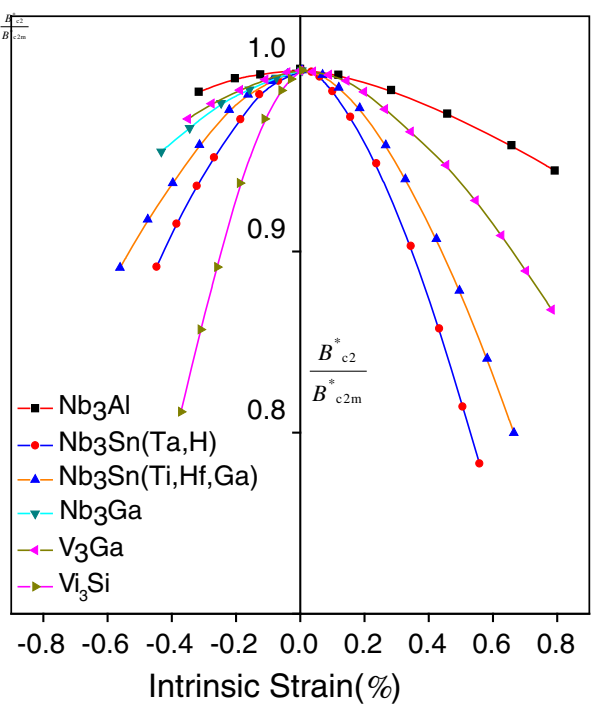

Fig. 5 Effect of uniaxial strain on the upper critical field of practical A15 superconductors

contributions into account made a new attempt to understand the stress effect. However, it did not consider the multi-scale structures at all.

\section{Discussion and conclusions}

Through surveying both the experimental and theoretical results on A15 compounds, we have provided a new multiscale point of view on the structure-properties relationships. It is obvious that there have been many phenomenological relations established. However, in most cases they are not well understood. The reason is that, in many cases, the passage from one smaller scale to larger scale is necessary, which remains a challenge in science. For A15 compounds, the question that which and how some information are transmitted from one scale to the other one has remained unclear. We suggest that the density of states may be the key parameter that survives the renormalization processes from smaller scale to the larger scale. This needs to be studied by more experimental and theoretical work. Superconductors have already been applied to many aspects of our modern society. A large-scale application to the high-speed train can be expected with the maturity of SCMaglev technique, which provides the fastest ground transportation system and saves more energy in comparison with the conventional Maglev system. Because of these large-scale applications, studies on superconductivity are both scientifically and economically crucially important.

Acknowledgments This work is financially supported by the Science Foundation for International Cooperation of Sichuan Province (2014HH0016), the Fundamental Research Funds for the Central
Universities (SWJTU2014: A0920502051113-10000), National Magnetic Confinement Fusion Science Program (2011GB112001).

Open Access This article is distributed under the terms of the Creative Commons Attribution License which permits any use, distribution, and reproduction in any medium, provided the original author(s) and the source are credited.

\section{References}

1. Hardy G, Hulm J (1953) Superconducting silicides and germanides. Phys Rev 89(4):883-884

2. Weger M, Goldberg B (1973) Lattice and electronic properties of the $\beta$-tungstens. Solid State Phys 28:1-177

3. Dew-Hughes D (1975) A-15 compounds: a review. Cryogenics 15(8):435-454

4. Godeke A (2006) A general scaling relation for the critical current density in $\mathrm{Nb}_{3} \mathrm{Sn}$. Supercond Sci Technol 19(10):100-116

5. Glowacki BA (1999) Niobium aluminide as a source of highcurrent superconductors. Intermetallics 7:117-140

6. Central Japan Railway Company Annual Report (2012), pp. $23-25$

7. Sweedler AR, Cox DE, Newkirk L (1975) Neutron irradiation effects in superconducting A-15 compounds. J Electron Mater 4(5):883-889

8. Brown BS, Blewitt TH, Wozniak DG et al (1975) Critical current changes in $\mathrm{Nb}_{3} \mathrm{Sn}$ irradiated with fast neutrons at $6 \mathrm{~K}$. J Appl Phys 46(12):5163-5168

9. Proceedings of the International Discussion Meeting on Radiation Effects on Superconductors, J Nucl Mat 1978, 72: 1-300

10. van Reuth EC, Waterstrat RM (1968) Atomic ordering in binary A15-type phases. Acta Cryst B24:168-196

11. Pande CS (1977) Effect of nuclear irradiation on the superconducting transition temperatures of A-15 materials. Solid State Commun 24(3):241-245

12. Paduani $C$ (2007) Electronic structure of the $A_{3} B$ compounds: $\mathrm{A}=\mathrm{Nb}: \mathrm{B}=\mathrm{Al}, \mathrm{Ga}, \mathrm{Ge}, \mathrm{Sn}$ and $\mathrm{In}$. Braz $\mathrm{J}$ Phys 37(3B): 1073-1079

13. Deng S, Simon A, Köhier J (1998) Superconductivity and chemical bonding in mercury. Angew Chem Int $\mathrm{Ed}$ 37(5):640-642

14. Ding YL, Deng SQ, Zhao Y The electronic structure of Nb3Al/ $\mathrm{Nb3Sn}$, a new test case for flat/steep band model of superconductivity. J Mod Transp, see this issue for the article of Ding et al.

15. Scanlan RM, Fietz WA, Koch EF (1975) Flux pinning centers in superconducting Nb3Sn. J Appl Phys 46(5):2244-2250

16. Shaw BJ (1976) Grain size and film thickness of Nb3Sn formed by solid-state diffusion in the range $650-800{ }^{\circ} \mathrm{C}$. J Appl Phys 47(5):2143-2146

17. Wadayama Y, Suzuki T, Aihara K et al (1991) Microstructural characterization of $\mathrm{Nb}_{3} \mathrm{Al}$ ultrafine multifilamentary superconducting wire by analytical electron microscopy. J Appl Phys 59(4):473-476

18. Banno N, Takeuchi T, Tsuchiya K (2012) Possible pining centers in transformation-processed $\mathrm{Nb}_{3} \mathrm{Al}$ superconductors. IEEE Trans Appl Supercond 22(3):6001504

19. Takeuchi T, Tsuchiya K, Saeda M (2010) Electron backscatter diffraction analysis of $\mathrm{Nb}_{3} \mathrm{Al}$ multifilamentary strands prepared by rapid heating, quenching and transformation annealing. Supercond Sci Technol 23:125001-125013

20. Takeuchi T, Tsuchiya K, Saeda M et al (2011) Electron-backscatter diffraction analysis of $\mathrm{Nb}_{3} \mathrm{Sn}$ and $\mathrm{Nb}_{3} \mathrm{Al}$ multifilaments. IEEE Trans on Appl Supercond 21(3):2541-2545 
21. Savitski EM, Bychkova MI, Baron VV et al (1976) Lattice instability of compound $\mathrm{Nb}_{3} \mathrm{Sn}$ in strained state. Phys Status Solidif A 37(2):165-167

22. Matthew CJ, Peter JL, David CL (2003) The influence of $\mathrm{Nb}_{3} \mathrm{Sn}$ strand geometry on filament breakage under bend strain as revealed by metallography. Supercond Sci Technol 16:1005-1011

23. Ekin JW (1984) Strain effects in superconducting compounds. Adv Cryo Eng 30:823-836

24. Taylor DM, Hampshire DP (2005) The scaling law for the strain dependence of the critical current density in Nb3Sn superconducting wires. Supercond Sci Technol 18:241-251
25. Markiewicz WD (2004) Invariant formulation of the strain dependence or the critical temperature $T_{\mathrm{c}}$ of $\mathrm{Nb}_{3} \mathrm{Sn}$ in a three term approximation. Cryogenics 44(11):895-908

26. Lim KC, Thompson JD, Webb GW (1983) Electronic density of states and $T_{\mathrm{c}}$ in $\mathrm{Nb}_{3} \mathrm{Sn}$ under pressure. Phys Rev B 27(5):2781-2787

27. Marzi GD, Morici L, Muzzi L et al (2013) Train sensitivity and superconducting properties of $\mathrm{Nb}_{3} \mathrm{Sn}$ from first principles calculations. J Phys 25(13):135702-135709 